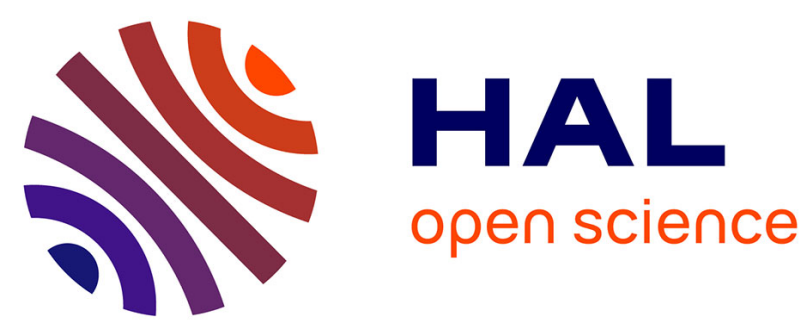

\title{
CARDS: A Mixed-Reality System for Collaborative Learning at School
}

Philippe Giraudeau, Alexis Olry, Joan Sol Roo, Stéphanie Fleck, David Bertolo, Robin Vivian, Martin Hachet

\section{> To cite this version:}

Philippe Giraudeau, Alexis Olry, Joan Sol Roo, Stéphanie Fleck, David Bertolo, et al.. CARDS: A Mixed-Reality System for Collaborative Learning at School. ACM ISS'19 - ACM International Conference on Interactive Surfaces and Spaces, Nov 2019, Daejeon, South Korea. pp.55-64, 10.1145/3343055.3359721. hal-02313463v3

\section{HAL Id: hal-02313463 \\ https://hal.inria.fr/hal-02313463v3}

Submitted on 1 Nov 2019

HAL is a multi-disciplinary open access archive for the deposit and dissemination of scientific research documents, whether they are published or not. The documents may come from teaching and research institutions in France or abroad, or from public or private research centers.
L'archive ouverte pluridisciplinaire HAL, est destinée au dépôt et à la diffusion de documents scientifiques de niveau recherche, publiés ou non, émanant des établissements d'enseignement et de recherche français ou étrangers, des laboratoires publics ou privés.

$$
\text { Copyright }
$$




\title{
CARDS: A Mixed-Reality System for Collaborative Learning at School
}

\author{
Philippe Giraudeau \\ Inria, Potioc * \\ Stephanie Fleck \\ Université de Lorraine, \\ PERSEUS $§$
}

Alexis Olry

Université de Lorraine, PERSEUS ${ }^{\dagger}$

\author{
David Bertolo \\ Université de Lorraine, \\ LCOMS $\mathbb{T}$
}

Joan Sol Roo
Inria, Potioc $¥$
Robin Vivian
Université de Lorraine, PERSEUS ॥

\section{Martin Hachet \\ Inria, Potioc **}

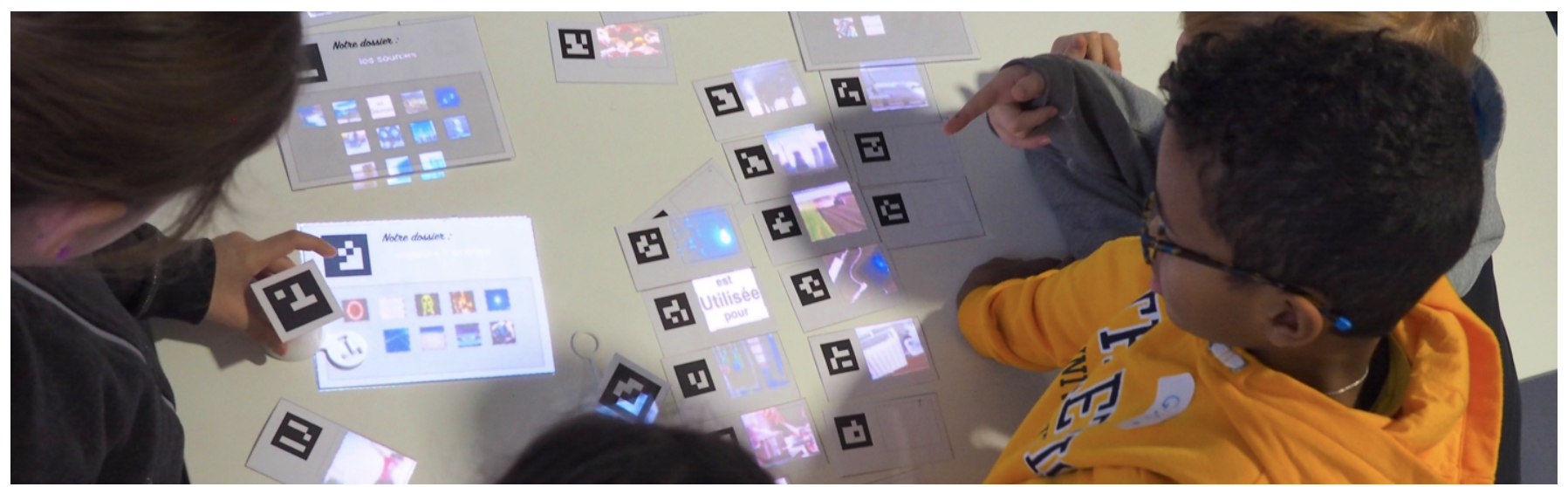

Figure 1. CARDS is a Mixed-Reality system targeted at Education where both physical and digital content are manipulated by several users in a seamless workspace.

\section{ABSTRACT}

Traditional computer systems based on the WIMP paradigm (Window, Icon, Menu, Pointer) have shown potential benefits at school (e.g. for web browsing). On the other hand, they are not well suited as soon as hands-on and collaborative activities are targeted. We present CARDS, a Mixed-Reality system that combines together physical and digital objects in a seamless workspace to foster active and collaborative learning. We describe the design process based on a participatory approach

\footnotetext{
*philippe.giraudeau@inria.fr

$\dagger$ alexis.olry@univ-lorraine.fr

†joan-sol.roo@inria.fr

§stephanie.fleck@univ-lorraine.fr

Idavid.bertolo@univ-lorraine.fr

"robin.vivian@univ-lorraine.fr

**martin.hachet@inria.fr
}

Permission to make digital or hard copies of all or part of this work for personal or classroom use is granted without fee provided that copies are not made or distributed for profit or commercial advantage and that copies bear this notice and the full citation on the first page. Copyrights for components of this work owned by others than ACM must be honored. Abstracting with credit is permitted. To copy otherwise, or republish, to post on servers or to redistribute to lists, requires prior specific permission and/or a fee. Request permissions from permissions@acm.org.

ISS '19, November 10-13, 2019, Deajon, Republic of Korea.

Copyright (C) 2019 Association of Computing Machinery.

ACM ISBN 978-1-4503-6891-9/19/11 ...\$15.00.

https://doi.org/10.1145/3343055.3359721 with researchers, teachers, and pupils. We then present and discuss the results of a user study that tends to show that CARDS has a good educational potential for the targeted activities.

\section{CCS Concepts}

-Human-Centered Computing $\rightarrow$ HCI design and evaluation methods; •Human-centered computing $\rightarrow$ Field studies;

\section{Author Keywords}

Augmented Reality, Spatial Augmented Reality, Tangible Interaction, Iterative Design, Education, Children-Computer Interaction

\section{INTRODUCTION}

Digital technologies have a major role in Education, as notably stated by the European commission [7, 26], which establishes digital technology in schools as one of the priorities for teaching, learning and developing 21 st century skills. Following this strong trend, numerous projects have introduced computers, tablets, and phones at school, and hundreds of dedicated applications are being developed. These applications generally rely on standard HCI paradigms, namely WIMP (Windows, Icons, Menus, Pointer) and touch-based adaptations. Such paradigms 
have shown to be useful for many tasks such as text editing or information gathering on the internet. On the other hand, it has been shown that they are limited for learning activities that involve practical work, and for collaborative activities that engage multiple participants in the classroom [31].

For such kinds of activities, ubiquitous approaches that take place in a three-dimensional space and foster spatial interactions and expressive representations seem to have a good potential [15]. In particular, digital approaches that are based on tangible interaction and augmented reality open new opportunities compared to more traditional desktop and tablet approaches [12]. Despite their potential, concrete implementations of such approaches at school remain rare. This invites us to propose fundamental and applied research to explore how tangible and mixed-reality approaches may serve the purpose of education, especially by promoting knowledge sharing, construction of concepts, and facilitation of social relationships. Following this general objective, we present a new interactive educational environment that is based on the manipulation of both physical and digital content (see Figure 1). This tool called CARDS for Collaborative Activities based on the Real and the Digital Superimposition, brings together hands-on and digital activities in a seamless workspace for collaborative learning at school.

As an example of pedagogical applications, in this work we target information sorting and mind-mapping building, where the participants can physically manipulate sheets of paper on which the digital content (e.g. texts, images, videos) is projected. As an illustrative scenario, the teacher can ask the pupils to discuss and create various categories (e.g. types of energy depending on their carbon impact). Thus, the pupils can sit around a standard school table on which CARDS has been set up. They can start working physically with the digital items that were provided by the teacher in a collective and autonomous way. CARDS has been designed by a research team composed of experts in Human-Computer Interaction (HCI), Ergonomics and Instructional Design (ID), and through several iteration cycles with children aged from 9 to 13, teachers, and educational practitioners. All along the design process, our objective has been to develop a tool that can be efficiently used at school by children, and that supports teachers' pedagogical sequences and collaborative learning dynamics.

The main contribution of this work is methodological in nature, where we describe the user-centered process that allowed us to propose a MR environment that fits the requirements of collective activities at school. We then describe the concrete technical implementation of this environment, as well as a first evaluation with pupils.

\section{RELATED WORK}

This project relies on the learning literature which supports the usage of the technical developments in the area of Mixed Reality and Tangible Interfaces in the context of education. Among the various interaction paradigms, the usage of augmented paper is one of the most promising.

\section{Learning and Reality-Based Interaction}

Learning is a cognitive process that allows the acquisition of new knowledge or the modification of existing one [21]. Among the factors that can influence the cognitive function of learning, three are particularly important: 1) attention, 2) engagement in the task (intrinsic motivation), stimulated by curiosity and 3 ) the feedback proposed by the activity (in our case, by the system) [9]. Bruner and Piaget's theory on learning stress the importance of multimodal interactions and in particular by using physical movements and embodiment [3]. Moreover, to optimize information retention, the material to be learned must be presented in such a way as to optimize the capacities of the working memory $[4,8]$. Learning can also be affected when the cognitive load [33] is too high. According to Sweller and his theory of Instructional Design [34], this charge can be described into three parts: 1) intrinsic cognitive load corresponding to the inherent complexity of the notion to learn; 2) extrinsic load that is generated by the presentation of information and 3) germane cognitive load corresponding to the load required by the cognitive system to store the information in memory. The latter can be optimized by limiting the extrinsic load and by providing a diversity of information presentation that allows both a better understanding and a development of cognitive schematics. Consequently, these aspects guided our technological choices.

Jacob [18] proposes the notion of Reality-Based Interaction (RBI) as a unifying framework linking different styles of interaction. They suggest that physical interactions (Naive Physics) have the potential to reduce the level of abstraction (extrinsic cognitive load) and facilitate learning through a more "intuitive" (meaning : facilitating through learning acquired since the user's early age) way to interact with the interface and its content [20]. This type of interface would also promote a more active engagement, through manual activities [20, 39], allowing the learning of more complex, abstract or very intangible notions [12]. In line with [32], transferring interactions with the digital world into the real one could scaffold cognition, i.e. represent an external resource that support cognition, Furthermore, this transfer mechanism can also support collaborative work as indicated by the theory of distributed cognition [22] by sharing our knowledge and workload between objects and users.

\section{SAR and TUI}

Although a large number of Augmented Reality (AR) interfaces have been designed for education (e.g., [6, 1]), we have favoured more ubiquitous approaches based on RBI, allowing direct interactions, supporting large spaces, as they facilitate sharing unified representations of the information.

Thus, we have based our work on Spatial Augmented Reality (SAR), which consists in augmenting physical objects by projecting computer-generated information onto their surfaces via a video-projector [24]. The concept of SAR was introduced by Raskar et al. [25], demonstrating the immersive potential of SAR. Yet, the first concept of augmentation through projection was presented by Wellner in 1993 [38] with its interface Digital Desk, allowing physical and virtual information to be manipulated similarly in the same space. 
One of the main benefits of SAR is that it allows its direct manipulation and spatialization by anchoring the digital content onto physical surfaces. Similarly, Tangible User Interfaces (TUIs) [29] focus on the integration and manipulating of digital information through physical artifacts. Given their common ground, it is not surprising that SAR and TUI are frequently used in combination.

\section{SAR, TUI, Learning :}

The coupling of these two paradigms is therefore particularly useful when creating hybrid environments where different users can interact around the same space, which can promote learning. A first example of this association with didactic capabilities was presented by Underkoffler et al. [35] where they used a video-projector to display on a tabletop a numerical simulation representing air flows that could be controlled by moving buildings miniatures. Later it was shown that this approach based on augmented physical manipulation can promote the understanding of complex and often abstract phenomena [12]. Still with the aim of facilitating the learning of complex concepts in a school context, Zufferey developed an augmented tabletop mixing SAR and TUI, called Tinker Lamp [40]. This hybrid environment is used in this scenario for the training of logistician apprentices. Tinker Lamp is a small-scale warehouse simulation environment where miniatures allow students to manipulate the warehouse layout to influence the simulation projected on the table. It has also been shown that this type of interface improves collaboration between students, increases the time spent exploring solutions during problem-solving tasks and, therefore, allows for better learning than a touch table [28]. Thinker Lamp was also used for creating concept map [10] in a collaborative learning scenario. In a Museum setting, Hornecker et al. [17, 14] have experimented with this type of interface dedicated to discovery and learning, showing that children were sensitive to the coupling between the shape of physical objects and their functioning within the system [16].

\section{Augmented Paper}

If the shape of objects in augmented environments is important to give meaning to interactions and their consequences, so is their everyday use. One example that makes sense in a school context is paper.

Wellner was the first to show the potential of this medium for SAR by using it as a projection medium [38]. This use was taken up by several interfaces dedicated to learning or creation. The concept of Dynamic Land [36] is completely based on affordance and the ability of paper to accommodate information, virtual or real. In Dynamic Land, each sheet of paper hosts a computer code that can be executed and attached to other codes to subsequently act on physical objects or an animation projected on the work surface. Thanks to their good accessibility, paper documents can then facilitate collaborative work by sharing the computer code or simulation projected on each sheet in plain view. Paper has also been used with augmented tabletops in schools for blind and visually impaired young people. This was notably the case for learning mobility and orientation with augmented tactile maps using projections and sound feedback [2]. The use of paper as tangible medium was preferred by the majority of blind and visually impaired students over 3D objects.

As it can be seen for the literature, the combination of spatial augmentation and tangible manipulations are excellent candidates to the creation of didactic interfaces, and paper if a promising support not only because of its affordances but also given its availability in classrooms. For these reasons, in this work we focused on this combination.

\section{REQUIREMENTS}

In order to produce specifications to guide our design, we conducted focus groups and observations in classrooms during more than 250 hours in a 6 months' period before development started.

\section{Focus Groups}

We performed 7 focus groups targeted on pedagogical and professional needs facing with collaborative learning. Feedback from teachers and education trainers $(n=18)$ provided pedagogical requirements. The most relevant are:

R1 Propose environments that are not too far from the realities of practice and compatible with the pedagogical use of paper-based resources (e.g., printed content, books, penand-paper work)

R2 Provide an interface that "makes you want to work", adaptable/compatible with the skill level of each learner to make pedagogical differentiation possible

R3 Provide systems that support two key cross-cutting competencies [23] for which children and teenagers need to be scaffolded: Competence. 1: Collectively and/or individually, process the information collected, organize them, store them in appropriate formats and format them ; Competence. 2: Work in a team, share tasks, engage in constructive dialogue

R4 Allow a very open degree of manipulation, move documents "freely" to provide some control over the actions to be taken, and make possible activities based on problem solving or investigating

R5 Highlight the need to sort documents and information in order to link and group them together. Provide a way to store this organisation (hierarchy and folders) as it.

Standard computers and tablets do not fulfill many of the identified requirements. This is also the case for digital white boards, or large touchscreens. These observed requirements tend to show that spatial augmented reality and tangible objects may be better suited than traditional digital technologies for many educational tasks at school.

\section{Observation in Classrooms}

In addition to focus groups, we conducted videotaped sessions at school. The collaborative dynamic observed in 36 different groups of 4 to 6 pupils from 2 primary and 1 secondary schools shows several important design considerations to take into account, particularly regarding its layout and tool availability. Main qualitative results indicate that the pupils systematically 


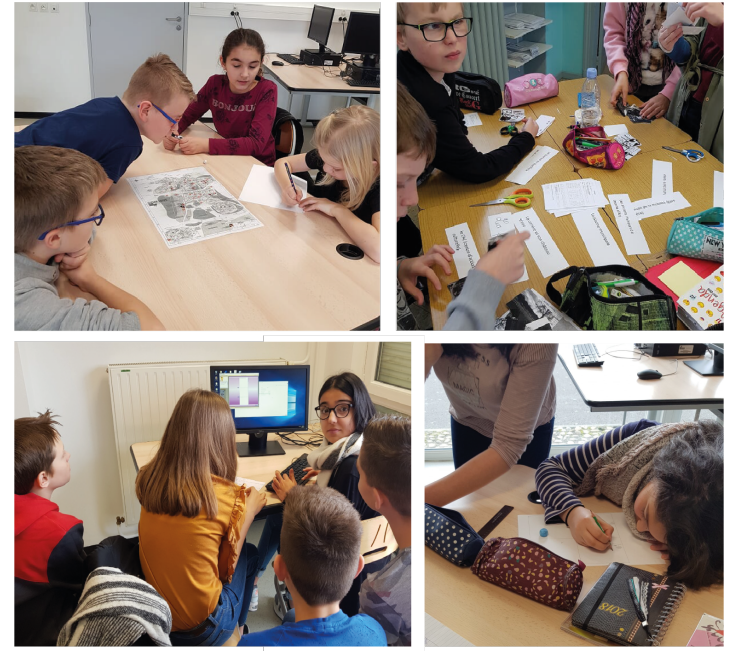

Figure 2. Examples of activities recorded during passive observations in class. Students work in groups on several tasks involving the use of different mediums (computer or tablet, paper/pencil).

displayed three types of roles: the operator, advisors and spectators when using limited tools (physical or digital). The student with the instrument to perform the task (pen, mouse, keyboard, etc.) take on the role of the operator. The student or students located in the visual proximity of the operator assume the role of advisor. The other pupils, far from the area of interaction, are placed in a spectator position, keeping them jobs out of the learning tasks.

Besides task distribution, the workspace layout and the size also shape the interaction: $360^{\circ}$ positioning of children around the documents limits the possibility for half of pupils to read and appropriate the content, and thus keeps children in their habitual individual work (see Figure 2). Consequently, 1) the workspace influence the position of pupils during collaborative activity with physical tools, either digital (during the observation, only WIMP) or physical (paper, pencils, etc.); 2) The availability of interaction tools represents a very strong constraint to the implementation of collaboration within the observed student groups and therefore to the learning of teamwork and task sharing.

By allowing a $180^{\circ}$ positioning in front of the learning supports and the availability of multiple tools, all content and learning tasks could become available to all children. In addition, the workspace also needs to be large enough to accommodate all children comfortably.

\section{ITERATIVE DESIGN}

Based on the focus groups and observational sessions, we conceived a hybrid system to support information classification and spatializations (see requirement R3). We titled the resulting system CARDS, which stand for Collaborative Activities based on the Real and the Digital Superposition (see Figure 1 ). This system was developed using an iterative process, performed in four stages, as illustrated in Figure 3. We conducted experiments at each stage, taking place either in schools or during scientific outreach events.
A total of 133 pupils between 9 and 13 years old participated during this 6-months iterative design. For each activity of the iterative design, in particular, cycle 2 , cycle 3 , an experimental protocol was defined, determining the activity to be carried out, the number of students per group and the data collected, including questionnaires and video recordings for future evaluations.

This section is organized as follows: For each cycle, we present the purpose of the cycle and the process by which we have implemented the targeted interactions, including the considered alternatives. Then, we describe the methodology, the results and we discuss them regarding that specific iteration. Finally, we present the qualitative results over all iterations.

\section{Cycle 1 - Data Physicalization}

The main purpose of the system is the ability offered to users to physically manipulate projected digital media as it could be done with physical ones. Supported media can be text, images, sounds or videos.

The basic atom of the environment takes the form of a rectangular paper card on which it is possible to project digital information on it (see Figure 1, and with a unique identifier marker (see section 5) printed on it. It is with this atom that users can physically manipulate any projected document following recommendation R1 (see section 3). The card also has its representation in the virtual environment allowing interactions with other objects and documents in the hybrid space.

We considered different form factors for the atom, ranging from a wooden token or smaller piece of paper that could only host the marker. In both cases, the media was projected around the object and not on top of it. We finally chose the card for its ability to host a projected media, enhancing the feeling of manipulating it, while reducing ambiguity regarding the association between the physical object and its media, particularly when physical objects are really close to each other. Then, we focused on the size, opting for images large enough $(6 \times 5 \mathrm{~cm})$ for direct manipulation by kids' hands without occlusions and small enough to allow several markers in the table.

Activity Setup: At the beginning of each activity, the virtual documents are randomly placed on the projection space and the cards have no documents attached.

Physical-Virtual association: A virtual document cannot be manipulated (e. g. enlarged, moved or edited) without having been attached to a physical object (card, folder, etc.). The association of a card with a media is achieved by overlapping them. The association is then made when the objects collide in the virtual space. The document will thus remain attached to the card for the duration of the handling.

These first functionalities allow the spatialization of the different media over the entire interaction space to perform document organization tasks.

\section{Observations and User Feedback}

The physicalization of virtual data was the subject of a presentation to two classes of pupils between 10 and 11 years old, during a scientific outreach's day. The objective of this 


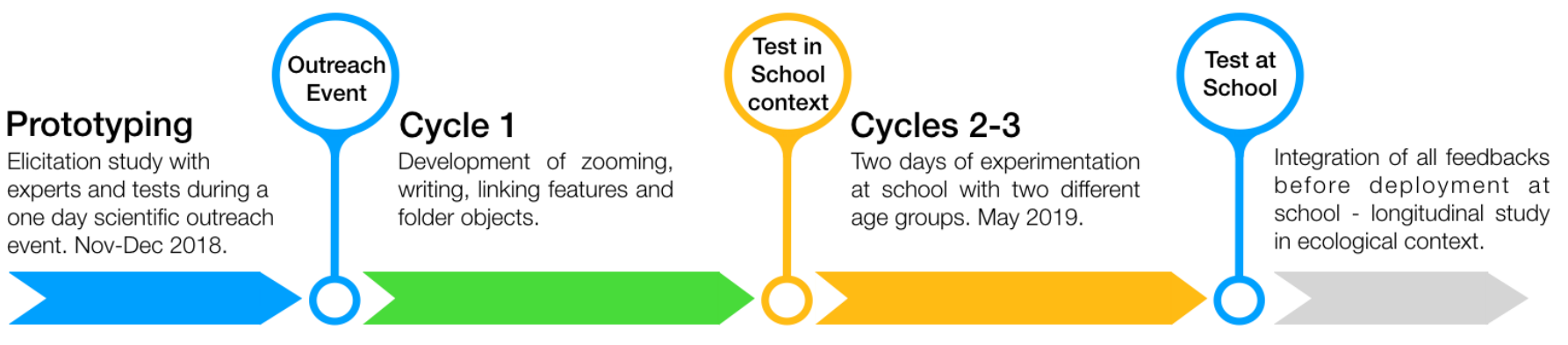

Figure 3. Overview of the iterative design process.

presentation was to check whether the pupils could understand how virtual documents were physicalized within a sheet of paper.

As an example exercise, a set of images corresponding to different modes of urban travel (i.e bike, bus, car, and so on ) had been placed on the table. The experimenters then asked the pupils to sort the images into groups. Once the task was completed, they were asked a question: "Do you find it complicated to manipulate the images with the cards?" they answered that they had no difficulty manipulating the images. However, the pupils were bothered by the instability of the detection system that made the cards "jump" or "blink". Beyond the need for stability required by the pupils, we observed that the resolution of the projector was a problem. Images seemed too pixelated without being small in size $(6 \times 5 \mathrm{~cm})$. Participants had difficulty to understand images and, as a result, to perform the requested sorting task. This was addressed by changing the projector (from $720 \mathrm{p}$ to $1080 \mathrm{p}$ ) and adding a temporal filter to the marker tracking.

\section{Cycle 2 - Editing, Prioritization and Collaboration}

The second cycle focused on the introduction of new mechanisms for managing the organization of documents within the workspace, as well as the addition of interactions to edit and view virtual documents (following requirements R3, R5 in Section 3). This iteration was the first in the cycle to be tested in a school context. For each of the manipulations explored in this cycle, diverse alternatives were considered, ranging from classical digital to pure tangible. The resulting solution utilizes a combination of both, as tangible tools support task distribution but are not particularly suited for usage in proximity with each other.

The manipulation mechanisms included in this cycle were:

Expand: In order to enlarge a document, we introduced a tangible token into the hybrid environment. The user with a physical magnifying glass pointer (see Figure 4-a) places the tip of the magnifying glass on the virtual document to increase its size.

Link: Creating links between objects is essential to perform a mind map task. In our system, linking two objects together is materialized by creating a line between these two objects. This line can be destroyed, named and customized, in particular by changing its color (see Section 4.2). During the first experiments around this functionality, several interactions were designed to link two objects together. The first way to create
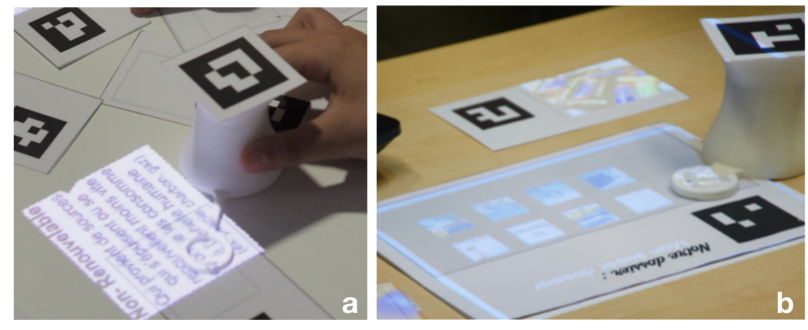

Figure 4. a) A physical pointer is used to expand a media. b) Another one is dedicated to the naming of the items.

and destroy the link was to bring the two objects close together. The same interaction was required to remove this link. This interaction was easily achievable but it tended to disrupt the spatialization of the documents on the table and prone to false positives when grouping objects. The second option, which was finally chosen, is to use an interactive pen that controls the computer cursor. To create the link, the user must touch the first object and then the second object to be linked in order to create the link. To delete a link between two objects, the user must "cut" the link with the digital pen.

Group: To group the items, the students have the possibility to aggregate the cards by spatializing them on the table and clustering them to create distinct categories. We have also explored techniques for grouping items, by introducing a "bubble" metaphor as done in bubble cluster [37]. However, this approach tends to clutter the space as soon as several items are manipulated, that is why we have introduced a new object, the Folder, that physically groups together a set of cards. Folders are objects composed of two pages, a front cover and a page to store the cards (see Figure 4-b). They preserve the same properties as the cards; they can be moved around freely and renamed. In addition, previews of the media stored in a Folder appear as thumbnail images on the front cover. When the folder is closed, the card group can be moved easily. It takes up less space in the hybrid workspace.

Edit: The editing and customization of documents is a strong request from teachers as part of educational sequences (see in particular Section 3). We first considered offering only the possibility for children to name or rename a media, in order to observe the use of this function and take into account user feedback. Thus, in the first version of the device, the naming of an item is done using the same gestural approach as the magnification interaction of an image. The user sets a specific physical pointer (see Figure 4-b). After placing the pointer on 
the media to be named, feedback in the form of a white outline appears. Starting from that point, the student uses a wireless keyboard. In addition to cards, folders and links can also be named.

\section{Observations and user feedback}

This first evaluation in the wild took place in a living lab, where a fifth-grade class (9-10 years old) and a 7 th grade class (12-13 years old) pupils were exposed to each of the features developed in this cycle. A semi-directed interview was conducted after the system manipulation. The children were thus invited to give their opinion on their experience with the latter. The semi-directed interview was divided into two parts. First, pupils were asked to write on Post-its their feelings about the objects they handled (cards, folders, physical pointers) and what they liked or disliked to avoid halo effect. Once they have written down their opinions, the experimenter asked them one by one, thus having the opportunity to ask for more details on the feelings of each student.

The results of these interviews can be divided into two parts: (1) user feedback on system usability and interactions, and (2) overall comments on the impression the system provides to users (e.g., stability, projection quality, projection area size).

Firstly, physical pointer zoom and edit have been identified as practical: "It's convenient", "it's easy to use" or "it's simple", another student finds that "Physical pointers are practical," but "we need to know the functions of objects to use them". This last comment invites us to work on the shape of the objects and their affordance. Regarding the files, the dominant comments pointed to a low stability, however, not disturbing: "The files were buggy, but it wasn't a problem to work with. Finally, the link creation with the interactive pen was noted as: "it's good to write with the computer and connect with the pen'. "The way the links were made was funny" or "The pen doesn't dirty the table". Overall, the proposed interactions were appreciated.

Although perceptible, the lack of stability did not seem to disturb the realization of the activity: "It's buggy, but you get used to it, you understand that's how it works." This comment is repeated by all groups of children. However, 5th grade pupils (11-12 years old) are more forgiving and do not take into account stability problems, unlike 6th grade (1011 years old) pupils, for whom this could be a problem when performing certain actions. This was the focus of the following iterations.

\section{Cycle 3 - Collaboration between objects and system sta- bility}

This last iteration opened the way to a new object that gathered the edit and zoom interactions into one new way to interact.

Inspect: The previous iteration introduces the interactions expand and edit. While the user feedback did not show any particular problems, during manipulations some manipulation problems appeared, especially during naming. In particular, two children were required to perform this task since the visual feedback (display of the current entry) was offset to the card level, making it difficult to read and confirm the action

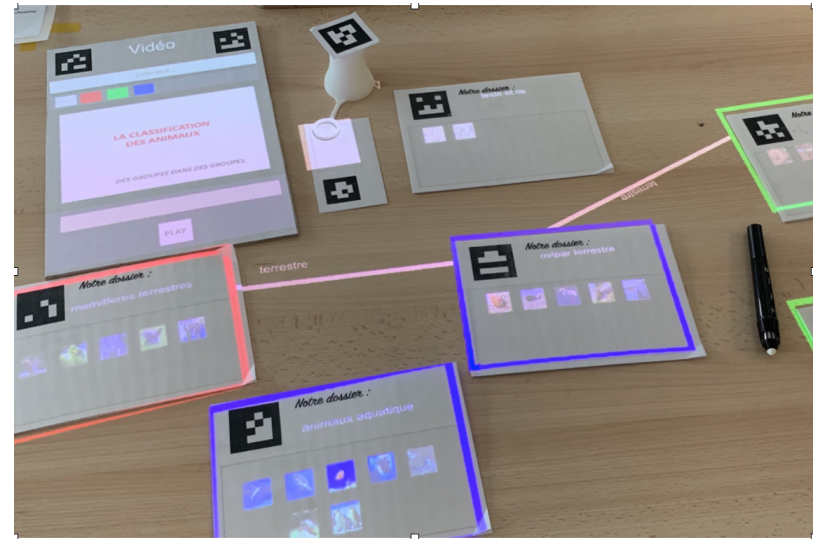

Figure 5. The tangible objects available in the latest version of the device. At the top left, the inspector with a map selected using a magnifying glass physical pointer, whose magnifying glass is positioned on the map. Folders are filled with maps and display previews of their contents. The links between the objects are named. Finally, the interactive pen is on the right side of the image.

performed. In addition, it was impossible for users to rename the cards while zooming in on them, mainly because of the occlusion generated by the two physical pointers surrounding the image. To compensate for these interaction errors, we have developed a new object with several functionalities. This object called Inspector (see figure 5), aims to replace the different editing actions by centralizing them within a single space. This object looking like rigid A4 paper allows children to rename a media, to know the type of document inspected (folder, link, videos...). To inspect an element, we kept the physical pointer magnifying glass (see Figure 5) and its interaction. We have also introduced the ability to view in detail, the media placed in a folder, without having to browse it manually as well as the ability to associate a color to a link, folder or card in order to facilitate the creation of groups of objects or to distinguish more easily between types of objects.

During this iteration, we did not ask for a return in the form of semi-structured interviews.

\section{Quantitative Results}

Throughout the participatory design cycle, quantitative evaluations were proposed to all participants, both teachers and children, through desirability questionnaires.

\section{Results of the Attrakdiff questionnaire}

The Attrakdiff questionnaire is based on a theoretical model using two dimensions: (i) the pragmatic qualities of the evaluated system, i.e. the ability to support the performance of the task (ii) the Hedonic qualities, namely, the potential of the system to be pleasant to use. The Attrakdiff presented to children is a simplified version of the original Attrakdiff, whose French version is validated, and which has already proven its suitability for the use of children [11]. Figure 6 compiles the feedback from teachers and children from the different sessions. One group was excluded due to extreme results: this group was in conflict with the adult supervisor. Overall, children and teachers believe that our system is desirable. We observe that through iterative cycles, children note the system 
as being more desirable (materialized by the arrow, see Figure $6)$. However, this observation is a trend but not a significant result. Figure 7 presents the results of the simplified Attrakdiff questionnaire in more detail. Globally, the pragmatic qualities are positively scored by the children (mean score: 1,36 - std: 1,049 with a scale between -3 and +3 ) and by the teachers ( mean score: 1,58 - std: 0,664). Regarding hedonist qualities the average is 1,87 (std: 0,91$)$ and 1,79 (std: 0,55$)$ for respectively children and adults.

The criteria evaluated, whether pragmatic, hedonistic or attractive, are positively rated. They particularly highlight the pragmatic qualities of "Simple" and "Practical" and the hedonistic qualities "captivating" and "top of the range". Furthermore, the last item presented in Figure 7 even though does not belong to the Attrakdiff, shows that children rated the system as motivating.

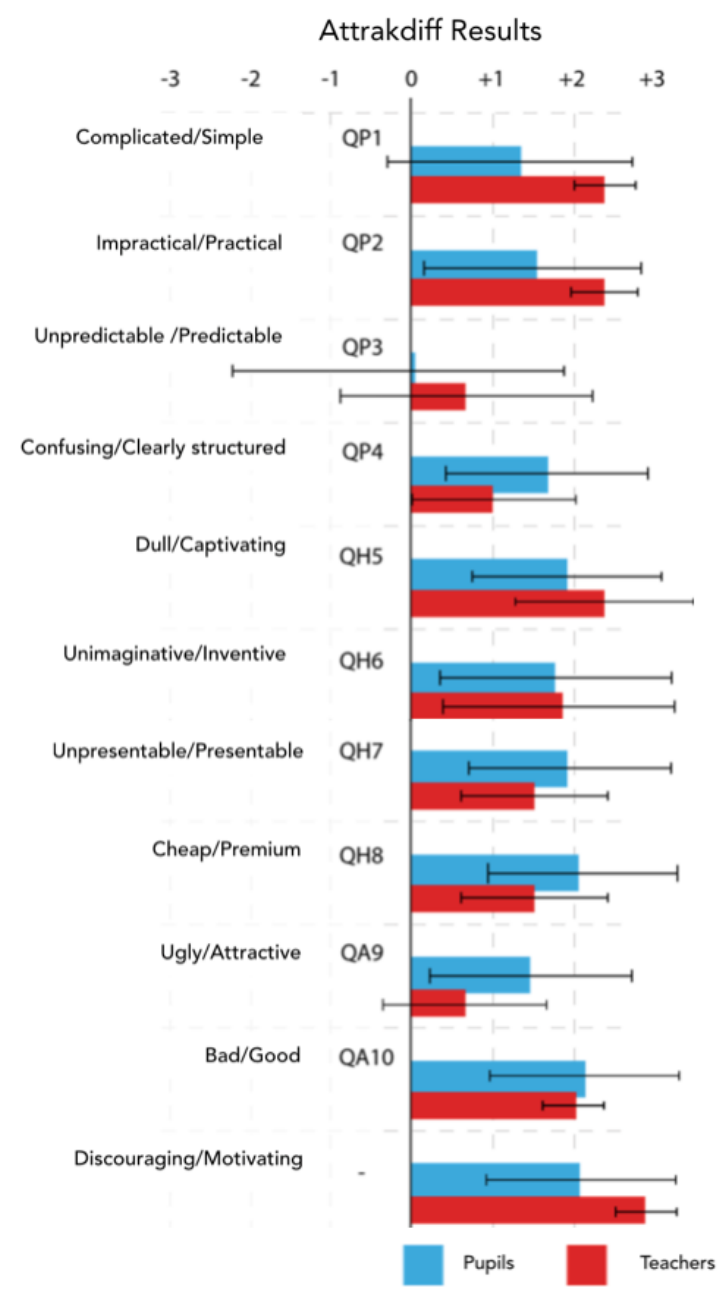

Figure 6. Results of the Attrakdiff questionnaire. The average value obtained at the hedonic scales are represented on the vertical axis and the average value at the pragmatic scale is represented on the horizontal axis. According to the scores obtained in both dimensions, the system was rated as desirable by all the groups interviewed.

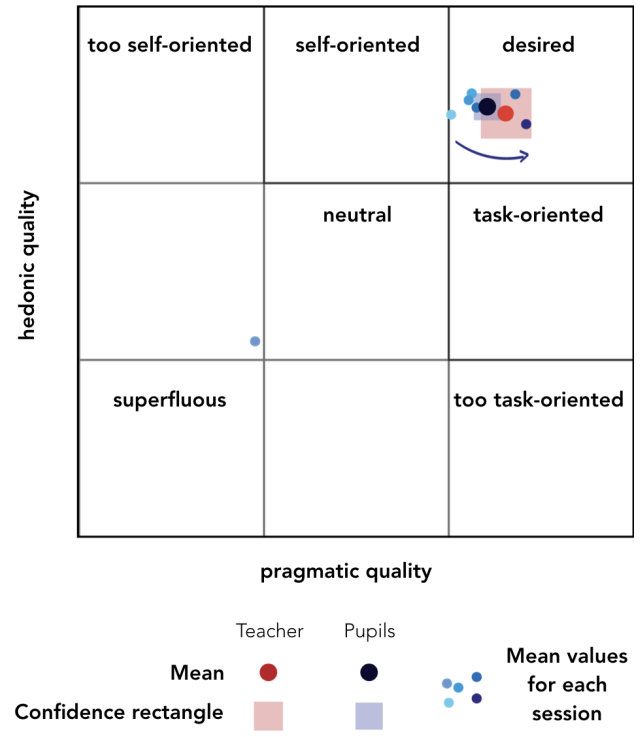

Figure 7. Results of the simplified Attrakdiff questionnaire after using the interface to perform an activity. "In general, I found the system." The evaluation is carried out according to a 7-point Likert discrimination scale (e.g. $(-3)$ Complicated/Simple $(+3))$; respectively coded from -3 to 3 or reversely. The attributes of Q1 to $4=$ Pragmatic Qualities, Q5 to 8 =Hedonist Qualities; $Q 9$ and $10=$ Overall Attractiveness of the system [19]. The last Item corresponds to the motivation and is not part of the simplified Attrakdiff. The arrow shows that children find the system more desirable through sessions.

These scores can be explained thanks to the literature, which points out the capacities of tangible and augmented interfaces to rely on the capacities acquired since the birth of users to handle objects (Naive Physics) [18]. Our design and the metaphors used such as cards (manipulation), folder (open/close) and links (use of a pen to "trace links") seek to minimize the gap between the handling of copy objects and their use in the hybrid environment. However, pragmatic qualities are impacted by the unpredictability of the system. Indeed, whether with regard to the population of teachers or students, all of them rate the stability of the system weakly (see figure 6, QP3), with significant variability between respondents. This lack of stability is linked to the difficulty to ensure a safe computer vision tracking of the manipulated objects. As a consequence, the system may sometimes respond differently from what the users had expected, which affects the user experience. Our current prototype is currently being improved to enhance the vision tracking part and to improve its general acceptability. It is also possible that the objects developed for CARDS are too focused on copying real objects. On this point, Hornecker et al [16] showed that children expected numerical increases to behave and react in the same way as imitated objects. This is a way to explore. However, despite these limitations, our current version of the prototype fulfill most of the requirements we have identified in Section 3), in particular R2 requirements.

\section{SYSTEM IMPLEMENTATION}

The device in its current version consists of a video-projector, a webcam, an interactive pen and a computer. An aluminium and wooden profile structure allows the video projector/camera to be attached to the edge of a table. The structure is designed to 


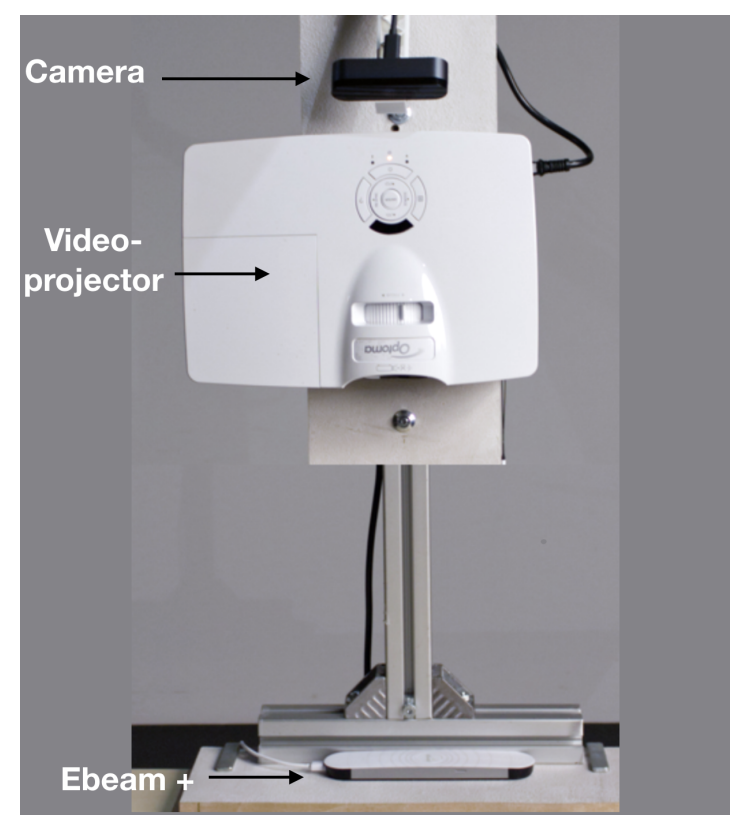

Figure 8. The aluminum and wooden profile structure supporting the projector and camera.

be rigid and lightweight, allowing the equipment to be moved without the need to calibrate the device before each use. For the first two iterations, the system had a short focal length Asus BM1R with a resolution of 720p and a brightness of 700 ANSI Lumens. In the last iteration, we changed the projector to a short focal length Optoma GT1080E with a resolution of $1080 \mathrm{p}$ and a brightness of 3000 ANSI Lumens. The camera is a Brio Logitech, allowing to provide a $720 \mathrm{p}$ video stream at 90 fps, connected in USB. The latency introduced by the webcam is about 180 Madam. The system uses an eBeam Edge+ to control the cursor and to interact with virtual objects.

The software part operates as a client-server architecture using $G R P C$ as a network protocol. The server processes the computer vision part, which is currently limited to the calibration and detection of fiduciary markers in space. It is developed in C++ and based on two software libraries OpenCV [5] and ArUco [27, 13].

The client deals with the interaction and graphic rendering part using the Unity $3 \mathrm{D}^{1}$ game engine. This architecture was selected in order to reduce tracking latency while supporting quick iteration over interaction techniques. It also allows the future support of distributed systems including mobile devices for orchestration.

The calibration of the projector/camera pair is performed offline. First, the camera is calibrated by using the OpenCV's calibrateCamera function. Then we defined a world origin (as a frame of reference) by placing a marker board (Aruco board) in order to locate markers in a 3D space rather than with respect to the camera. It is also mandatory for the last state of the calibration.

\footnotetext{
${ }^{1}$ Unity 3D https://unity.com
}

The third step consists in finding the intrinsic and extrinsic parameters of the video-projector using a manual method (see [30] by matching reference pixels with their 3D position using the already calibrated camera. These steps have to be done at least once when building the hardware system. Once the system is assembled and calibrated, users no longer need to calibrate the system (except for falls or major shocks).

\section{DISCUSSION}

The final version of CARDS is then composed of paper slips that act as physical proxies for digital elements such as text, videos and pictures. Theses cards can be freely manipulated in the environment allowing grouping, organizing and selecting information based on the inherent capacity of the system to spatialize items. We also introduced augmented folders that can store cards and display thumbnails onto their cover page. Folder enables users to reduce the amount of items on the tabletop and work with higher information. In order to create or delete links between objects on the table, we use an interactive pen. The links, as any object on the table, can be edited (change its name or color). The Inspector allows the pupils to visualize a closer view of the content that is stored into cards or folders and edit it. It can also customize links by changing the label or colors associated to it.

\section{What we have learnt during iterative design with children}

Developing hybrid systems for out-of-laboratory usage is particularly difficult, especially when users are pupils in a school environment. Nevertheless, during a 6-month period of experimentation, we were able to measure the potential of a hybrid system to transform the way teachers and pupils use digital tools at school. The potential of hybrid interfaces in school such as CARDS is high, but it is important to highlight a few points when designing interactions. Thus, the greatest advantage of hybrid interfaces lies in their ability to copy real interactions and simplify the entry into the task. However, copying the interactions that could be achieved in the real world also has a drawback. When interactions are too straightforward, students begin to forget that these interactions are in fact virtual and it creates a situation where children initiate unstable state that disturbs the immersion in the system. On the other side, the introduction of the inspector, as it looks more like a virtual object (button, input box), limits such troubles. Students do not have to automate the fact of having to click a button again if the desired interaction did not take place. Thus, a potential recommendation to the community would be to pay attention to the design of objects and interactions that can be achieved in the same way in the real world and in the hybrid environment.

The use of iterative design in a school context allowed us to understand the end user who is in our case very different from an adult. We were able to observe differences in children's behaviour between scientific outreach sessions and sessions in the school context (at school or in the living lab). Throughout iterative cycles, we observed that the use and the perception of CARDS by children changed depending on their age. Although the age range of the population is quite small, younger children had a more restrictive use of the environment but were more easily bothered by some behaviours not anticipated 
by the system or by the children. On the contrary, older pupils were able to accomplish the task by going beyond the limitations. Other ecological factors such as the composition of the groups of pupils, the time of day and the location of the test also influence the pupils' perception of the interface. Children are more focused on the task at school than on a scientific outreach event and tend to be more serious on what we asked. This kind of difference impacted the quality of feedback. We also figure out than letting students try the system without help from the experimenters provided an opportunity to observe how children understand interactions and the philosophy behind it. Those behaviours provided complementary feedback with semi-directed interviews or direct questions asked during manipulation.

\section{CONCLUSION AND DIRECTION TO FUTURE WORK}

In this paper, we have presented CARDS, an interactive system whose objective is to promote collaborative learning at school through physical manipulation of augmented cards. This system was designed on the basis of theoretical considerations from the literature, practical considerations related to the school environment in which it is intended to be used, and an iterative design process guided by experiments with pupils and teachers. This approach has allowed us to design a new interactive system that appears to be globally desired by users. On the other hand, the current version of the prototype still needs to be stabilized to allow better usability. This usability will be evaluated in a school context, without the presence of the experimenters, after a new iteration phase dedicated to stabilizing the system. Thereafter, we will seek to develop aspects related to the orchestration and regulation in the classroom to enable real pedagogical activities to be carried out in the school.

\section{ACKNOWLEDGMENTS}

This work is part of the e-TAC project financially supported by the Caisse des Dépôts et Consignations as part of the eFRAN projects. We would like to thank Solène Lambert for her investment. We also would like to thank all the teachers and children for their contribution in the project.

\section{REFERENCES}

[1] Murat Akçayır and Gökçe Akçayır. 2017. Advantages and challenges associated with augmented reality for education: A systematic review of the literature. Educational Research Review 20 (2017), 1-11.

[2] Jérémy Albouys-Perrois, Jérémy Laviole, Carine Briant, and Anke Brock. 2018. Towards a multisensory augmented reality map for blind and low vision people: A participatory design approach. In CHI 2018, Conference on Human Factors in Computing Systems. ACM, Montréal, Canada.

https://hal-enac . archives-ouvertes . fr/hal-01801116

[3] Alissa N Antle. 2007. The CTI framework: informing the design of tangible systems for children. In Proceedings of the 1st international conference on Tangible and embedded interaction. ACM, 195-202.

[4] Alan D Baddeley. 1997. Human memory: Theory and practice. Psychology Press.
[5] G. Bradski. 2000. The OpenCV Library. Dr. Dobb's Journal of Software Tools (2000).

[6] Peng Chen, Xiaolin Liu, Wei Cheng, and Ronghuai Huang. 2017. A review of using Augmented Reality in Education from 2011 to 2016. In Innovations in smart learning. Springer, 13-18.

[7] European Commission. 2009. Europeans, Science and Technology. Report. http://ec.europa.eu/public_ opinion/archives/ebs/ebs_224_report_en.pdf

[8] Nelson Cowan. 2001. Metatheory of storage capacity limits. Behavioral and brain sciences 24, 1 (2001), 154-176.

[9] Stanislas Dehaene. 2014. Collège de France Courses Cognitive Psychologie : Fondements cognitifs des apprentissages scolaires. (2014), 36.

[10] Son Do-Lenh, Patrick Jermann, Amanda Legge, Guillaume Zufferey, and Pierre Dillenbourg. 2012. TinkerLamp 2.0: designing and evaluating orchestration technologies for the classroom. In European Conference on Technology Enhanced Learning. Springer, 65-78.

[11] Stéphanie Fleck, Charlotte Baraudon, Jérémy Frey, Thibault Lainé, and Martin Hachet. 2017. 'Teegi, He's so cute" : Example of pedagogical potential testing of an interactive tangible interface for children at school. In 29ème conférence francophone sur l'Interaction Homme-Machine, AFIHM (Ed.). ACM, Poitiers, France, 12 p. DOI : http://dx. doi .org/10.1145/3132129.3132143

[12] Stéphanie Fleck and Martin Hachet. 2016. Making tangible the intangible: Hybridization of the real and the virtual to enhance learning of abstract phenomena. Frontiers in ICT 3 (Nov. 2016), 30. DOI : http://dx.doi.org/10.3389/fict.2016.00030

[13] Sergio Garrido-Jurado, Rafael Muñoz-Salinas, Francisco Madrid-Cuevas, and Rafael Medina-Carnicer. 2015. Generation of fiducial marker dictionaries using Mixed Integer Linear Programming. Pattern Recognition 51 (10 2015). DOI :

http://dx.doi.org/10.1016/j.patcog.2015.09.023

[14] E. Hornecker. 2008. "I don't understand it either, but it is cool" - visitor interactions with a multi-touch table in a museum. In 2008 3rd IEEE International Workshop on Horizontal Interactive Human Computer Systems. 113-120. DOI : http://dx. doi .org/10.1109/TABLETOP. 2008.4660193

[15] Eva Hornecker and Jacob Buur. 2006. Getting a grip on tangible interaction: a framework on physical space and social interaction. In Proceedings of the SIGCHI conference on Human Factors in computing systems. ACM, 437-446.

[16] Eva Hornecker and Andreas Dünser. 2008. Of pages and paddles: Children's expectations and mistaken interactions with physical-digital tools. Interacting with Computers 21, 1-2 (2008), 95-107. 
[17] Eva Hornecker and Matthias Stifter. 2006. Learning from Interactive Museum Installations About Interaction Design for Public Settings. In Proceedings of the 18th Australia Conference on Computer-Human Interaction: Design: Activities, Artefacts and Environments (OZCHI '06). ACM, New York, NY, USA, 135-142. DOI : http://dx.doi.org/10.1145/1228175.1228201

[18] Robert JK Jacob, Audrey Girouard, Leanne M Hirshfield, Michael S Horn, Orit Shaer, Erin Treacy Solovey, and Jamie Zigelbaum. 2008. Reality-based interaction: a framework for post-WIMP interfaces. In Proceedings of the SIGCHI conference on Human factors in computing systems. ACM, 201-210.

[19] Carine Lallemand, Vincent Koenig, Guillaume Gronier, and Romain Martin. 2015. Création et validation d'une version française du questionnaire AttrakDiff pour l'évaluation de l'expérience utilisateur des systèmes interactifs. Revue Européenne de Psychologie Appliquée/European Review of Applied Psychology 65 (09 2015), 239-252. DOI :

http://dx.doi.org/10.1016/j. erap. 2015.08.002

[20] Paul Marshall. 2007. Do tangible interfaces enhance learning? Proceedings of the 1st international conference on Tangible and embedded interaction - TEI '07 (2007), 163.

[21] Richard E Mayer. 2005. Cognitive theory of multimedia learning. The Cambridge handbook of multimedia learning 3148 (2005).

[22] Donald A Norman. 1991. Cognitive artifacts. Designing interaction: Psychology at the human-computer interface 1 (1991), 17-38.

[23] Pierre Rabardel and Renan Samurçay. 2006. De l'apprentissage par les artefacts à l'apprentissage médiatisé par les instruments. Sujets, activités, environnements. Approches transversales (2006), 31-60.

[24] Ramesh Raskar and Kok-Lim Low. 2001. Interacting with spatially augmented reality. In Proceedings of the lst international conference on Computer graphics, virtual reality and visualisation. ACM, 101-108.

[25] Ramesh Raskar, Greg Welch, and Henry Fuchs. 1998. Spatially augmented reality. In First IEEE Workshop on Augmented Reality (IWAR'98). 11-20.

[26] European Commission Eurydice report. 2013. Education and Training in Europe 2020: respons from the EU member states. Report.

[27] Francisco Romero Ramirez, Rafael Muñoz-Salinas, and Rafael Medina-Carnicer. 2018. Speeded Up Detection of Squared Fiducial Markers. Image and Vision Computing 76 (06 2018). DOI :

http://dx.doi.org/10.1016/j.imavis.2018.05.004
[28] Bertrand Schneider, Patrick Jermann, Guillaume Zufferey, and Pierre Dillenbourg. 2011. Benefits of a tangible interface for collaborative learning and interaction. IEEE Transactions on Learning Technologies 4, 3 (2011), 222-232.

[29] Orit Shaer, Eva Hornecker, and others. 2010. Tangible user interfaces: past, present, and future directions. Foundations and Trends ${ }^{\circledR}$ in Human-Computer Interaction 3, 1-2 (2010), 4-137.

[30] Joan Sol Roo. 2017. One Reality Augmenting the human experience through the combination of physical and digital worlds. Theses. Universite Bordeaux. https://hal . archives-ouvertes.fr/tel-01702720

[31] Danae Stanton and HR 2003 Neale. 2003. The effects of multiple mice on children's talk and interaction. Journal of Computer Assisted Learning 19, 2 (2003), 229-238.

[32] John Sutton, Celia B Harris, Paul G Keil, and Amanda J Barnier. 2010. The psychology of memory, extended cognition, and socially distributed remembering. Phenomenology and the cognitive sciences 9, 4 (2010), 521-560.

[33] John Sweller. 1994. Cognitive load theory, learning difficulty, and instructional design. Learning and instruction 4, 4 (1994), 295-312.

[34] J Sweller. 1999. Instructional Design in Technical Areas. Australian Education Review, No. 43. PCS Data Processing. Inc. USA (1999).

[35] John Underkoffler and Hiroshi Ishii. 1999. Urp: a luminous-tangible workbench for urban planning and design. In Proceedings of the SIGCHI conference on Human Factors in Computing Systems. ACM, 386-393.

[36] Bret Victor. Dynamicland. (????). https://dynamicland.org/

[37] Nayuko Watanabe, Motoi Washida, and Takeo Igarashi. 2007. Bubble clusters: an interface for manipulating spatial aggregation of graphical objects. In Proceedings of the 20th annual ACM symposium on User interface software and technology. ACM, 173-182.

[38] Pierre Wellner, Wendy Mackay, and Rich Gold. 1993. Computer-augmented environments: back to the real world. Commun. ACM 36, 7 (1993), 24-27.

[39] Oren Zuckerman, Saeed Arida, and Mitchel Resnick. 2005. Extending tangible interfaces for education: digital montessori-inspired manipulatives. In Proceedings of the SIGCHI conference on Human factors in computing systems. ACM, 859-868.

[40] Guillaume Zufferey, Patrick Jermann, Aurélien Lucchi, and Pierre Dillenbourg. 2009. TinkerSheets: using paper forms to control and visualize tangible simulations. In Proceedings of the 3rd international Conference on Tangible and Embedded interaction. ACM, 377-384. 